\title{
A Combinatorial Characterization of Resolution Width
}

\author{
Albert Atserias * \\ Universitat Politècnica de Catalunya \\ Barcelona, Spain
}

\author{
Víctor Dalmau ${ }^{\dagger}$ \\ Universitat Pompeu Fabra \\ Barcelona, Spain
}

September 2, 2005

\begin{abstract}
We provide a characterization of the resolution width introduced in the context of propositional proof complexity in terms of the existential pebble game introduced in the context of finite model theory. The characterization is tight and purely combinatorial. Our first application of this result is a surprising proof that the minimum space of refuting a 3-CNF formula is always bounded from below by the minimum width of refuting it (minus 3). This solves a well-known open problem. The second application is the unification of several width lower bound arguments, and a new width lower bound for the dense linear order principle. Since we also show that this principle has resolution refutations of polynomial size, this provides yet another example showing that the relationship between size and width cannot be made subpolynomial.
\end{abstract}

\section{Introduction}

Resolution is one of the most popular proof systems for propositional logic. Since Haken [18] proved an exponential lower bound for the smallest resolution proofs of the pigeonhole principle, its strength has been studied in depth. The focus has been put in two related directions: (1) proving strong lower bounds for interesting tautologies arising from combinatorial principles $[29,11,7,9,4,24,26]$, and (2) the study of the complexity of finding resolution proofs $[7,9,3,6]$. This research is still ongoing, and it seems that further study in both directions is necessary for a better understanding of the power of resolution.

An important step towards the understanding of the strength of resolution in a unified way was made by Ben-Sasson and Wigderson [9] with the introduction of the width measure. The width of a resolution refutation is the size of the largest clause in the refutation. The main result of Ben-Sasson and Wigderson, building upon the work of Clegg, Edmonds and Impagliazzo [12] and Beame and Pitassi [7], is the following: if a 3-CNF formula with $n$ variables has a resolution refutation of size $S$, then it has a resolution refutation of width $O(\sqrt{n \log S})$. This interesting result relates the size with the width in a form that is suitable to prove size lower bounds. Indeed, if the minimal width of refuting $F$ is $w$, then every resolution refutation of $F$ requires size $2^{\Omega\left(w^{2} / n\right)}$. Equipped with this result, Ben-Sasson and Wigderson not only re-derived all previously known lower bounds for resolution in an elegant and unified way, but also showed that resolution

\footnotetext{
${ }^{*}$ Supported in part by CICYT TIN2004-04343 and by the European Commission through the RTN COMBSTRU HPRNCT2002-00278.

${ }^{\dagger}$ Research partially supported by the MEC under the program "Ramon y Cajal", grants TIC 2002-04470-C03 and TIC 200204019-C03, the EU PASCAL Network of Excellence, IST-2002-506778, and the MODNET Marie Curie Research Training Network, MRTN-CT-2004-512234.
} 
is automatizable in subexponential time by an extremely simple dynamic programming algorithm. We should notice, however, that the size-width relationship of Ben-Sasson and Wigderson has shown insufficient to prove size lower bounds for some interesting cases such as the weak pigeonhole principle. In fact, Bonet and Galesi [10] proved that the size-width trade-off is essentially tight, and therefore the technique cannot be applied to it. Indeed, the 3-CNF encoding of the weak pigeonhole principle with $m^{2}$ pigeons and $m$ holes has $n=O\left(m^{3}\right)$ variables, but the best width lower bound that can be proved is $w=\Omega(m)$. Note that the lower bound $2^{\Omega\left(w^{2} / n\right)}$ from the size-width trade-off is just trivial in this case. The problem about the weak pigeonhole principle was finally solved by Raz [24] (see also [25]) using a completely different technique.

Our goal in this paper is to establish a tight connection between the resolution width of Ben-Sasson and Wigderson, and the existential $k$-pebble game, first introduced by Kolaitis and Vardi $[19,20]$ in the context of finite model theory. Research in this direction was initiated by Atserias [5] in the study of the descriptive complexity of properties that certify unsatisfiability of random CNF formulas.

Ehrenfeucht-Fraïssé games is the generic name for the combinatorial two-player games that characterize expressibility in several logics, including first-order logic, second-order logic, and fragments of infinitary logic (see [14]). Among these, we encounter the existential $k$-pebble game, introduced by Kolaitis and Vardi $[19,20]$ to analyze the expressive power of Datalog, a well-known query language in database theory. The game is played between two players, the Spoiler and the Duplicator, on two relational structures $\mathbf{A}$ and $\mathbf{B}$ over the same vocabulary. Each player has a set of $k$ pebbles numbered $\{1, \ldots, k\}$. In each round of the game, the Spoiler can make one of two different moves: either he places a free pebble over an element of the domain of $\mathbf{A}$, or he removes a pebble from a pebbled element of $\mathbf{A}$. To each move of the Spoiler, the Duplicator must respond by placing her corresponding pebble over an element of $\mathbf{B}$, or removing her corresponding pebble from $\mathbf{B}$ respectively. If the Spoiler reaches a round in which the set of pairs of pebbled elements is not a partial homomorphism between $\mathbf{A}$ and $\mathbf{B}$, then he wins the game. Otherwise, we say that the Duplicator wins the game.

The crucial fact that relates pebble games to resolution width is the observation, first pointed out by Feder and Vardi [16], that the satisfiability problem of an $r$-CNF formula $F$ can be identified with the homomorphism problem on relational structures: given two finite relational structures $\mathbf{A}$ and $\mathbf{B}$ over the same vocabulary, is there a homomorphism from $\mathbf{A}$ to $\mathbf{B}$ ? Informally, the structure $\mathbf{A}$ represents the variables and the clauses of $F$, the structure $\mathbf{B}$ represents the truth-values $\{0,1\}$ and the combination of them that are valid assignments for the clauses, and the homomorphisms from $\mathbf{A}$ to $\mathbf{B}$ are precisely the assignments of variables to truth-values satisfying all the clauses of $F$. Using this reformulation, we show that the concepts of resolution width and pebble games are intimately related. More specifically, we prove that $F$ has a resolution refutation of width $k$ if and only if the Spoiler wins the existential $(k+1)$-pebble game on $\mathbf{A}$ and $\mathbf{B}$. Thus, existential $k$-pebble games provide a purely combinatorial characterization of resolution width.

The new characterization allows us to re-derive, in a uniform way, essentially all known width lower bounds. Moreover, an increase of insight generally reverts in the acquisition of new results. This case is no exception. Our first application of the combinatorial characterization is a surprising result relating the space and the width in resolution. The space measure was introduced by Esteban and Torán [15] (see also [1]). Intuitively, the minimal resolution space of refuting a CNF formula $F$ is the number of clauses that are required to be kept in a blackboard (memory) if we insist that the refutation must be self-contained. This measure is referred to as the clause space by Alekhnovich, Ben-Sasson, Razborov and Wigderson [1]. Strong space lower bounds were proved in the literature for well-known tautologies such as the pigeonhole principle [28, 1], Tseitin tautologies [28, 1], graph tautologies [1], and random formulas [8] to cite some. Our new result is that the minimum space of refuting an $r$-CNF formula is always bigger than the minimum width of refuting $F$ minus $r-1$. In symbols, $s(F) \geq w(F)-r+1$. Thus, for $r$-CNF formulas with small $r$, space lower bounds follow at once from width lower bounds. We remark that Torán [28] already obtained this result for the restricted case of tree-like resolution, but the general case remained open since then. Our 
result confirms the conjecture of Ben-Sasson and Galesi [8] and answers questions posed by Esteban and Torán [15], and Alekhnovich, Ben-Sasson, Razborov and Wigderson [1].

The second application of our result is a new width lower bound. We consider the dense linear order principle $D L O_{n}$, suggested by Urquhart and used by Riis [27], and stating that no finite linear order is dense. We show that every resolution refutation of $D L O_{n}$ requires width at least $n / 3$, where $n$ is the number of elements of the linear order. We want to make the point that the significance of this result is not so much the width lower bound itself, but rather, the techniques that are involved and that we discuss next.

Most of the tautologies studied in the literature, including $D L O_{n}$, have large initial width. Consequently, in order to get meaningful width lower bounds it is necessary to convert them into equivalent and short (generally 3-CNF) formulas in a preliminary step. Unfortunately, it is usually the case that the resulting formula looses some of the intuitive appeal of the principle it expresses. Furthermore, in a width lower bound proof, dealing with the auxiliary variables is usually simple but cumbersome and laborious. To simplify this situation we define a variant of the pebble game, called extended pebble game, that can be played directly over formulas with large clauses and that hides all the technical details, such as the process of dividing large clauses, the introduction of auxiliary variables and its treatment, inside the proof of its main property. In particular, the width lower bound for the $D L O_{n}$ is obtained this way. We complete the picture about $D L O_{n}$ by showing that it has polynomial-size resolution proofs. Thus, the $D L O_{n}$ principle provides a new example requiring large width but having small resolution proofs (see $[10,3,6]$ for further discussion on this).

\section{Preliminaries}

Let $V$ be a set of propositional variables. A literal is a variable or the negation of a variable. A clause is a set of literals. If a clause has at most $r$ literals, we call it an $r$-clause. An $r$-CNF formula is a set of $r$ clauses. Alternatively, clauses may be viewed as disjunctions of literals, and CNF formulas may be viewed as conjunctions of clauses. For a clause $C$, let $\operatorname{var}(C)$ be the set of variables that appear in it. A partial truth assignment to $V$ is any function $f: V^{\prime} \rightarrow\{0,1\}$ where $V^{\prime} \subseteq V$. We say that $f$ falsifies a clause $C$ if it sets all literals from $C$ to 0 . Dually, we say that $f$ satisfies $C$ if it sets some literal from $C$ to 1 . In all other cases we say that $f$ leaves $C$ undecided. Resolution is a refutation system that works with clauses. The only rule is the so-called resolution rule:

$$
\frac{C \cup\{x\} \quad D \cup\{\neg x\}}{C \cup D}
$$

where $C$ and $D$ are arbitrary clauses and $x$ is a variable. A resolution derivation from a set of initial clauses $F$ is a sequence of clauses $C_{1}, \ldots, C_{m}$, each of which is either a clause from $F$, or follows by the resolution rule from two previous clauses in the sequence. We say that $C_{1}, \ldots, C_{m}$ is a resolution derivation of $C_{m}$ from $F$. If $C_{m}$ is the empty clause \{\} , we say that $C_{1}, \ldots, C_{m}$ is a refutation of $F$. The width of a clause is the number of literals in it. The width of a derivation is the maximum of the widths of its clauses.

Let $L=\left\{R_{1}, \ldots, R_{m}\right\}$ be a finite relational language, that is, a finite set of relation symbols with an associated arity. An $L$-structure is a tuple $\mathbf{A}=\left(A, R_{1}^{\mathbf{A}}, \ldots, R_{m}^{\mathbf{A}}\right)$ where $A$ is a set, called the universe, and $R_{i}^{\mathbf{A}} \subseteq A^{k_{i}}$ is a $k_{i}$-ary relation on $A$, where $k_{i}$ is the arity of $R_{i}$. Let $\mathbf{A}=\left(A, R_{1}^{\mathbf{A}}, \ldots, R_{m}^{\mathbf{A}}\right)$ and $\mathbf{B}=\left(B, R_{1}^{\mathbf{B}}, \ldots, R_{m}^{\mathbf{B}}\right)$ be $L$-structures. A partial homomorphism from $\mathbf{A}$ to $\mathbf{B}$ is any function $f: A^{\prime} \rightarrow B$, where $A^{\prime} \subseteq A$, such that $f$ defines an homomorphism from the substructure of $\mathbf{A}$ with domain $A^{\prime}$ to the structure $\mathbf{B}$. In other words, $f$ is a function such that for every relation symbol $R \in L$ of arity $s$ and $a_{1}, \ldots, a_{s} \in A^{\prime}$, if $\left(a_{1}, \ldots, a_{s}\right) \in R^{\mathbf{A}}$ then $\left(f\left(a_{1}\right), \ldots, f\left(a_{s}\right)\right) \in R^{\mathbf{B}}$. If $f$ and $g$ are partial homomorphisms, we say that $g$ extends $f$, denoted by $f \subseteq g$, if $\operatorname{Dom}(f) \subseteq \operatorname{Dom}(g)$ and $f(a)=g(a)$ for every $a \in \operatorname{Dom}(f)$. If $f \subseteq g$, we also say that $f$ is the projection of $g$ to $\operatorname{Dom}(f)$. 
The existential $k$-pebble game on $\mathbf{A}$ and $\mathbf{B}$ is played by two players: the Spoiler and the Duplicator. Each player has a set of $k$ pebbles numbered $\{1, \ldots, k\}$. In each round of the game, the Spoiler can make one of two different moves: either he places a free pebble over an element of the domain of $\mathbf{A}$, or he removes a pebble from a pebbled element of $\mathbf{A}$. To each move of the Spoiler, the Duplicator must respond by placing her corresponding pebble over an element of $\mathbf{B}$, or removing her corresponding pebble from $\mathbf{B}$ respectively. If the Spoiler reaches a round in which the set of pairs of pebbled elements is not a partial homomorphism between $\mathbf{A}$ and $\mathbf{B}$, then he wins the game (note that if two different pebbles are placed on the same element of $\mathbf{A}$ but the two corresponding pebbles are placed over different elements of $\mathbf{B}$, then the set of pairs does not define a partial homomorphism). Otherwise, we say that the Duplicator wins the game. The next definition formalizes this intuitive discussion:

Definition 1 ([19, 20]) Let $L$ be a finite relational language and let $\mathbf{A}$ and $\mathbf{B}$ be $L$-structures. We say that the Duplicator wins the k-pebble game on $\mathbf{A}$ and $\mathbf{B}$ if there is a nonempty family $\mathcal{H}$ of partial homomorphisms from $\mathbf{A}$ to $\mathbf{B}$ such that

(i) If $f \in \mathcal{H}$, then $|\operatorname{Dom}(f)| \leq k$.

(ii) If $f \in \mathcal{H}$ and $g \subseteq f$, then $g \in \mathcal{H}$.

(iii) If $f \in \mathcal{H}$, $|\operatorname{Dom}(f)|<k$, and $a \in A$, then there is some $g \in \mathcal{H}$ such that $f \subseteq g$ and $a \in \operatorname{Dom}(g)$.

We say that $\mathcal{H}$ is a winning strategy for the Duplicator.

Intuitively, each partial homomorphism $g \in \mathcal{H}$ is a winning position for the Duplicator in the game. Let us mention that the existential $k$-pebble game is known to characterize definability in the existential positive $k$-variable fragment of infinitary logic $\exists \mathcal{L}_{\infty \omega \omega}^{k}$, that is, the logic that is obtained by closing the set of atomic formulas over the variables $x_{1}, \ldots, x_{k}$ under infinitary conjunctions, infinitary disjunctions, and existential quantification of a variable in $x_{1}, \ldots, x_{k}$. Note that variables may be re-used. Although we will not use it in this paper, let us state the result that links existential $k$-pebble games and definability in $\exists \mathcal{L}_{\infty \omega}^{k}$.

Theorem 1 [19, 20] Let $L$ be a finite relational language and let $\mathbf{A}$ and $\mathbf{B}$ be $L$-structures. Then, the Duplicator wins the existential k-pebble game on $\mathbf{A}$ and $\mathbf{B}$ if and only if every $\exists \mathcal{L}_{\infty \omega^{-}}^{k}$-sentence that holds in $\mathbf{A}$ also holds in $\mathbf{B}$.

The $k$-variable fragments of infinitary logics have played a crucial role in the development of finite model theory (see [17] for a good survey).

\section{Combinatorial Characterization as Games}

It is well known that $r$-CNF formulas may be encoded as finite relational structures. Indeed, let $L=$ $\left\{P_{0}, P_{1}, \ldots, P_{r}\right\}$ be the finite relational language that consists of $r+1$ relations of arity $r$ each. An $r$-CNF formula $F$ over the propositional variables $v_{1}, \ldots, v_{n}$ is encoded as an $L$-structure $\mathbf{M}(F)$ as follows. The domain of $\mathbf{M}(F)$ is the set of variables $\left\{v_{1}, \ldots, v_{n}\right\}$. For each $s \in\{0, \ldots, r\}$, the relation $P_{s}$ encodes the set of clauses of $F$ with $s$ negated variables. More precisely, the interpretation of $P_{s}$ consists of all $r$-tuples of the form

$$
\left(v_{i_{1}}, \ldots, v_{i_{s}}, v_{i_{s+1}}, \ldots, v_{i_{r}}\right) \in\left\{v_{1}, \ldots, v_{n}\right\}^{r}
$$

such that $\left\{\neg v_{i_{1}}, \ldots, \neg v_{i_{s}}, v_{i_{s+1}}, \ldots, v_{i_{r}}\right\}$ is a clause of $F$. Note that we do not require the variables to be different, so our clauses have at most $r$ literals, and not necessarily exactly $r$. Next we define a particular $r$-CNF formula $T_{r}$ whose encoding $\mathbf{M}\left(T_{r}\right)$ is of our interest. The clauses of $T_{r}$ are all the $r$-clauses on the variables $v_{0}$ and $v_{1}$ that are satisfied by the truth assignment that maps $v_{0}$ to 0 , and $v_{1}$ to 1 . 
We will consider the particular case of the existential $k$-pebble game that is played on the structures $\mathbf{M}(F)$ and $\mathbf{M}\left(T_{r}\right)$. Observe that each partial homomorphism from $\mathbf{M}(F)$ to $\mathbf{M}\left(T_{r}\right)$ may be viewed as a partial truth assignment to the variables of $F$ that does not falsify any clause from $F$. Since this is central to the later development, let us state it as a lemma.

Lemma 1 Let $F$ be an $r$-CNF formula, and let $h$ be a partial homomorphism from $\mathbf{M}(F)$ to $\mathbf{M}\left(T_{r}\right)$. Then $h$ is also a partial truth assignment that does not falsify any clause from $F$.

Proof: Suppose $h$ falsifies a clause $C$. Let $s$ be the number of negated literals in $C$ and let $\left(v_{1}, \ldots, v_{r}\right) \in$ $P_{s}^{\mathbf{M}(F)}$ be the $r$-tuple encoding $C$ in $\mathbf{M}(F)$. Since $h$ falsifies $C$, we must have $h\left(v_{1}\right)=\cdots=h\left(v_{s}\right)=1$ and $h\left(v_{s+1}\right)=\cdots=h\left(v_{r}\right)=0$. But the tuple $(1, \ldots, 1,0, \ldots, 0)$ with $s$ ones and $r-s$ zeros does not belong to $P_{s}^{\mathbf{M}\left(T_{r}\right)}$ because it does not satisfy the clause $\neg v_{0} \vee \cdots \vee \neg v_{0} \vee v_{1} \vee \cdots \vee v_{1}$ with $s$ negated literals and $r-s$ positive literals. We conclude that $h$ is not a partial homomorphism; contradiction.

Thus, the existential $k$-pebble game on $\mathbf{M}(F)$ and $\mathbf{M}\left(T_{r}\right)$ may be reformulated as follows.

Definition 2 Let $F$ be an $r$-CNF formula. We say that the Duplicator wins the Boolean existential $k$-pebble game on $F$ if there is a nonempty family $\mathcal{H}$ of partial truth assignments that do not falsify any clause from F such that

(i) If $f \in \mathcal{H}$, then $|\operatorname{Dom}(f)| \leq k$.

(ii) If $f \in \mathcal{H}$ and $g \subseteq f$, then $g \in \mathcal{H}$.

(iii) If $f \in \mathcal{H}$, $|\operatorname{Dom}(f)|<k$, and $x$ is a variable, then there is some $g \in \mathcal{H}$ such that $f \subseteq g$ and $x \in \operatorname{Dom}(g)$.

We say that $\mathcal{H}$ is a winning strategy for the Duplicator.

We stress on the fact that this definition is only a particular case of the definition of winning strategy for the existential $k$-pebble game defined in Section 2.

Lemma 2 If there is no resolution refutation of $F$ of width $k$, then the Duplicator wins the Boolean existential $(k+1)$-pebble game on $F$.

Proof: Let $\mathcal{C}=\left\{C_{1}, \ldots, C_{m}\right\}$ be the set of all clauses having a resolution derivation from $F$ of width at most $k$. Let $\mathcal{H}$ be the set of all partial truth assignments with domain of size at most $k+1$ that do not falsify any clause in $\mathcal{C}$. We will see that $\mathcal{H}$ is a winning strategy. Clearly $\mathcal{H}$ is not empty since it contains the partial truth assignment with empty domain (note that $C_{1}, \ldots, C_{m}$ does not contain the empty clause). Clearly, $\mathcal{H}$ is closed under projections. Now, let $f$ be any partial truth assignment in $\mathcal{H}$ with $|\operatorname{Dom}(f)| \leq k$, and let $x$ be any variable not in $\operatorname{Dom}(f)$. Let us assume that there does not exist a valid extension of $f$ to $x$ in $\mathcal{H}$. In this case let $C \in \mathcal{C}$ be the clause falsified by the extension of $f$ that maps $x$ to 0 . Clearly $C=C^{\prime} \cup\{x\}$ since otherwise $f$ would falsify $C$. Analogously there exits some $D \in \mathcal{C}$ of the form $D^{\prime} \cup\{\neg x\}$ that is falsified by the extension of $f$ that maps $x$ to 1 . Note now that $\operatorname{var}\left(C^{\prime} \cup D^{\prime}\right) \subseteq \operatorname{Dom}(f)$, so $C^{\prime} \cup D^{\prime}$ has width at most $k$ and belongs to $\mathcal{C}$. In particular, $f$ does not falsify it, so it cannot falsify both $C^{\prime} \cup\{x\}$ and $D^{\prime} \cup\{\neg x\}$; contradiction.

Lemma 3 If the Duplicator wins the Boolean existential $(k+1)$-pebble game on $F$, then there is no resolution refutation of $F$ of width $k$. 
Proof: Let $\mathcal{H}$ be a winning strategy for the Duplicator for the existential $(k+1)$-pebble game on $F$. We will show by induction in the resolution proof of width $k$ that no partial truth assignment in $\mathcal{H}$ falsifies a clause of the proof. Thus, the proof cannot be a refutation. The statement is clearly satisfied by the initial clauses since we are dealing with partial truth assignments that do not falsify any clause of $F$. Let $C \cup\{x\}$ and $D \cup\{\neg x\}$ be clauses of the proof, and let $C \cup D$ be the result of applying the resolution rule. Let $f$ be any partial truth assignment in $\mathcal{H}$. If the domain of $f$ does not include all the variables in $C \cup D$ then we are done since it cannot falsify it. Otherwise consider the projection $g$ of $f$ to the variables in $C \cup D$. We will show that $g$ (and hence $f$ ) does not falsify $C \cup D$. Since the width of $C \cup D$ is at most $k$, the domain of $g$ has size at most $k$. Therefore, there exists some extension $h$ of $g$ to $x$ such that $h$ is in $\mathcal{H}$. By induction hypothesis $h$ does not falsify any of $C \cup\{x\}$ and $D \cup\{\neg x\}$. Consequently, since $h$ falsifies $x$ or $\neg x, h$ cannot falsify $C \cup D$ either.

Combining these two lemmas we obtain the main result of this section. We say that the Spoiler wins the Boolean existential $k$-pebble game on $F$ if the Duplicator does not win the Boolean existential $k$-pebble game on $F$.

Theorem 2 Let $F$ be an $r$-CNF formula. Then, $F$ has a resolution refutation of width $k$ if and only if the Spoiler wins the Boolean existential $(k+1)$-pebble game on $F$.

We note that the Boolean existential $k$-pebble game does not talk about resolution at all. Thus, this provides a purely combinatorial characterization of resolution width.

\section{Application: Width versus Space}

In this section we show that the resolution space introduced by Esteban and Torán [15] and by Alekhnovich, Ben-Sasson, Razborov and Wigderson [1] is tightly related to the width. Indeed, for an $r$-CNF formula $F$, the minimal space $s(F)$ of refuting $F$, is always bounded from below by $w(F)-r$, where $w(F)$ is the minimal width of refuting $F$. This solves an open problem in $[28,1,8]$.

We start with some definitions. Let $F$ be an $r$-CNF formula. A configuration is a set of clauses. A sequence of configurations $\mathcal{C}_{0}, \mathcal{C}_{1}, \ldots, \mathcal{C}_{r}$ is a self-contained resolution proof if $\mathcal{C}_{0}=\emptyset$ and for $i>0$, the configuration $\mathcal{C}_{i}$ is obtained from $\mathcal{C}_{i-1}$ by one of the following rules:

(i) Axiom Download: $\mathcal{C}_{i}=\mathcal{C}_{i-1} \cup\{C\}$ for some $C \in F$,

(ii) Erasure: $\mathcal{C}_{i}=\mathcal{C}_{i-1}-\{C\}$ for some $C \in \mathcal{C}_{i-1}$,

(iii) Inference: $\mathcal{C}_{i}=\mathcal{C}_{i-1} \cup\{C\}$ for some $C$ that is obtained from an application of the resolution rule on two clauses from $\mathcal{C}_{i-1}$.

The space of a self-contained resolution proof $\mathcal{C}_{0}, \ldots, \mathcal{C}_{r}$ is the maximum of $\left|\mathcal{C}_{i}\right|$ for $i=0, \ldots, r$. A selfcontained resolution refutation is a self-contained resolution proof whose last configuration is $\{\square\}$. The minimal space of refuting an unsatisfiable formula $F$, denoted by $s(F)$, is the minimal space of all selfcontained resolution refutations of $F$. We will need the following easy lemma.

Lemma 4 (Locality Lemma [1]) Let $f$ be a partial truth assignment and let $\mathcal{C}$ be a set of clauses. If $f$ satisfies $\mathcal{C}$, then there exists a restriction $g \subseteq f$ such that $|\operatorname{Dom}(g)| \leq|\mathcal{C}|$ and $g$ still satisfies $\mathcal{C}$.

Proof: For every $C \in \mathcal{C}$, let $l_{C} \in C$ be a literal that is satisfied by $f$. For every $l_{C}$, let $x_{C}$ be the underlying variable. Finally, let $g$ be the projection of $f$ to $\left\{x_{C}: C \in \mathcal{C}\right\}$. 
Lemma 5 Let $F$ be an unsatisfiable $r-C N F$ formula, and let $k \geq 1$. If the Duplicator wins the Boolean existential $(k+r-1)$-pebble game on $F$, then the minimal space of refuting $F$ is at least $k$.

Proof: Let $\mathcal{H}$ be a winning strategy for the Duplicator in the existential $(k+r-1)$-pebble game on $F$. We show that if $\mathcal{C}_{0}, \ldots, \mathcal{C}_{m}$ is a self-contained resolution proof of space less than $k$, then every $\mathcal{C}_{i}$ is satisfiable. This will prove that $F$ cannot have a resolution refutation of space less than $k$. We build, by induction on $i$, a sequence of partial truth assignments $f_{i} \in \mathcal{H}$ such that $f_{i}$ satisfies $\mathcal{C}_{i}$ and $\left|\operatorname{Dom}\left(f_{i}\right)\right| \leq\left|\mathcal{C}_{i}\right|$. In the following, let $s_{i}=\left|\mathcal{C}_{i}\right|$. Let $f_{0}=\emptyset$. In order to define $f_{i}$ for $i>0$, suppose that $f_{i-1}$ has already been defined. We consider the three possible scenarios for $\mathcal{C}_{i}$. Case 1: $\mathcal{C}_{i}=\mathcal{C}_{i-1} \cup\{C\}$ by an axiom download for $C \in F$. Let $f \in \mathcal{H}$ be an extension of $f_{i-1}$ such that all variables in $C$ are in $\operatorname{Dom}(f)$. Since $\left|\operatorname{Dom}\left(f_{i-1}\right)\right| \leq s_{i-1}<k$, $f_{i-1} \in \mathcal{H}$ and $C$ is an $r$-clause, such an $f$ exists in $\mathcal{H}$. Moreover, since $f$ does not falsify any clause from $F$, and since all variables in $C$ are defined, $f$ satisfies $C$. Therefore, $f$ satisfies $\mathcal{C}_{i}$. Now, by the Locality Lemma, there exists some restriction $g \subseteq f$ such that $|\operatorname{Dom}(g)| \leq s_{i}$ and still $g$ satisfies $\mathcal{C}_{i}$ and belongs to $\mathcal{H}$. Let $f_{i}=g$. Case 2: $\mathcal{C}_{i}=\mathcal{C}_{i-1} \cup\{C\}$ by an inference. In this case set $f_{i}=f_{i-1}$. The soundness of the resolution rule guarantees that $f_{i}$ satisfies $\mathcal{C}_{i}$. Of course, $\left|\operatorname{Dom}\left(f_{i}\right)\right| \leq s_{i-1} \leq s_{i}$ and $f_{i} \in \mathcal{H}$. Case 3: $\mathcal{C}_{i}=\mathcal{C}_{i-1}-\{C\}$ by a memory erasure. Obviously, $f_{i-1}$ still satisfies $\mathcal{C}_{i}$ since it satisfies $\mathcal{C}_{i-1}$. Now, by the Locality Lemma, there is a restriction $g$ of $f_{i-1}$ such that $|\operatorname{Dom}(g)| \leq s_{i}$ and still $g$ satisfies $\mathcal{C}_{i}$ and belongs to $\mathcal{H}$. Let $f_{i}=g$.

Theorem 3 Let $F$ be an unsatisfiable $r$-CNF formula. Then, $s(F) \geq w(F)-r+1$ where $s(F)$ is the minimal space of refuting $F$ in resolution, and $w(F)$ is the minimal width of refuting $F$ in resolution.

Proof: Let $w=w(F)$. Then there is no resolution refutation of width $w-1$ of $F$. By Theorem 2, the Duplicator wins the Boolean existential $w$-pebble game on $F$. But then, by Lemma 5, the minimal space of refuting $F$ is at least $w-r+1$. Hence $s(F) \geq w-r+1$.

We note that this theorem can be used to derive space lower bounds for all formulas for which width lower bounds are known such as the pigeonhole principle, Tseitin formulas, random formulas, and so on.

\section{Application: Unified Width Lower Bounds}

The new characterization of the width can be used to obtain width lower bounds in a simpler and unified way. For CNF formulas whose clauses are already small, the width lower bound is obtained directly by exhibiting a winning strategy for the Duplicator. We illustrate this point with the encoding of the pigeonhole principle into an unsatisfiable 3-CNF formula by means of auxiliary variables (the so-called standard nondeterministic extension).

We will consider the 3 -CNF formula $E P H P_{n}^{n+1}$ encoding the negation of the pigeonhole principle. For every $i \in\{1, \ldots, n+1\}$ and $j \in\{1, \ldots, n\}$, let $p_{i, j}$ be a propositional variable meaning that pigeon $i$ sits in hole $j$. For every $i \in\{1, \ldots, n+1\}$ and $j \in\{0, \ldots, n\}$, let $y_{i, j}$ be a new propositional variable. The following 3-CNF formula $E P_{i}$ expresses that pigeon $i$ sits in some hole:

$$
E P_{i} \equiv \neg y_{i, 0} \wedge \bigwedge_{j=1}^{n}\left(y_{i, j-1} \vee p_{i, j} \vee \neg y_{i, j}\right) \wedge y_{i, n}
$$

Finally, the 3-CNF formula $E P H P_{n}^{n+1}$ expressing the negation of the pigeonhole principle is the conjunction of all $E P_{i}$ and all clauses $H_{k}^{i, j} \equiv \neg p_{i, k} \vee \neg p_{j, k}$ for $i, j \in\{1, \ldots, n+1\}, i \neq j$ and $k \in\{1, \ldots, n\}$.

Lemma 6 The Duplicator wins the Boolean existential n-pebble game on EPHP ${ }_{n}^{n+1}$. 
Proof: Let $\mathcal{B}$ be the set of all one-to-one partial functions from $\{1, \ldots, n+1\}$ into $\{1, \ldots, n\}$. For every $a \in \mathcal{B}$, define a partial truth assignment $h_{a}$ as follows:

(i) $h_{a}\left(p_{i, j}\right)=1$ if $a(i)$ is defined and $a(i)=j$,

(ii) $h_{a}\left(p_{i, j}\right)=0$ if $a(i)$ is defined and $a(i) \neq j$,

(iii) $h_{a}\left(y_{i, j}\right)=0$ if $a(i)$ is defined and $a(i)>j$,

(iv) $h_{a}\left(y_{i, j}\right)=1$ if $a(i)$ is defined and $a(i) \leq j$.

Let $\mathcal{F}=\left\{h_{a}: a \in \mathcal{B}\right\}$, and let $\mathcal{H}$ be the set of restrictions of assignments of $\mathcal{F}$ to all sets of at most $n$ variables. It is straightforward to check that $\mathcal{H}$ is a winning strategy for the Duplicator: property (i) is met because, by definition, all assignments in $\mathcal{F}$ have at most $n$ variables in the domain, property (ii) is met by definition as well, and property (iii) is met because if $h \subseteq h_{a}$ has at most $n-1$ variables in its domain, then there is an empty hole.

We claim that all width lower bounds in the literature can be easily re-derived by exhibiting a winning strategy for the Duplicator. For example, [5] provided a winning strategy for the Duplicator for random formulas, and thus width lower bounds are also derived for them.

Our next twist is an attempt to systematize the use of the extension variables such as the $y_{i, j}$ 's in $E P H P_{n}^{n+1}$. The point is that we would like to play games on CNF formulas with arbitrarily long clauses, and derive meaningful width lower bounds for their standard non-deterministic extensions. For an arbitrary CNF formula $F$ without any restriction on the length of its clauses, let us define an equivalent $r$-CNF formula for $r \geq 3$. Such a formula is called the standard non-deterministic extension of $F$ in [1]. For every clause $C$ of length at most $r$, let $E_{r}(C)=C$. For every clause $C=\left\{l_{1}, \ldots, l_{w}\right\}$ of length $w>r$, let $y_{C, 0}, \ldots, y_{C, w}$ be a collection of new variables. Then we define $E_{r}(C)$ as follows:

$$
E_{r}(C) \equiv \neg y_{C, 0} \wedge \bigwedge_{j=1}^{w}\left(y_{C, j-1} \vee l_{i} \vee \neg y_{C, j}\right) \wedge y_{C, w}
$$

Then, $E_{r}(F)$ is the conjunction of all $E_{r}(C)$. Note that $E_{r}(F)$ is now an $r$-CNF formula and it is unsatisfiable if and only if $F$ is.

The aim of the following definitions is to formalize a variation on the existential $k$-pebble game that is tailored for the non-deterministic extensions that we just introduced. Let $F$ be a CNF formula without any restriction on the length of its clauses. Let $V$ be the set of propositional variables of $F$. An extended partial truth assignment $a$ is a pair $(D, f)$ where $D \subseteq V \times(F \cup\{1\})$ and $f$ is partial truth assignment. Moreover, if $D=\left\{\left(x_{1}, C_{1}\right), \ldots,\left(x_{r}, C_{r}\right)\right\}$, then $\operatorname{Dom}(f)=\left\{x_{1}, \ldots, x_{r}\right\}$ and clause $C_{i}$ is satisfied by setting $x_{i}$ to $f\left(x_{i}\right)$ (note that 1 is always satisfied). If $a=(D, f)$ and $b=(E, g)$ are extended partial truth assignments, we say that $b$ is an extension of $a$, denoted by $a \subseteq b$, if $D \subseteq E$ and $f \subseteq g$. We also say that $a$ is a projection of $b$. We say that an extended partial truth assignment $(D, f)$ does not falsify a clause if $f$ does not falsify it.

Definition 3 We say that the Duplicator wins the extended $(r, k)$-game on $F$ if there is a nonempty family $\mathcal{A}$ of extended partial truth assignments that do not falsify any clause of $F$ such that

(i) If $(D, f) \in \mathcal{A}$, then $|D| \leq k$.

(ii) If $(D, f) \in \mathcal{A}$ and $E \subseteq D$, then there is some $g \subseteq f$ such that $(E, g) \in \mathcal{A}$.

(iii) If $(D, f) \in \mathcal{A},|D|<k$, and $x \in V$, then there is some $g$ such that $f \subseteq g$ and $(D \cup\{(x, 1)\}, g) \in \mathcal{A}$. 
(iv) If $(D, f) \in \mathcal{A},|D|<k$, and $C \in F$ has length at least $r+1$, then there is some $g$ and some $x \in V$ such that $f \subseteq g$ and $(D \cup\{(x, C)\}, g) \in \mathcal{A}$.

The main result about this new game is the following lemma.

Lemma 7 If the Duplicator wins the extended $(r, k)$-game on $F$, then the Duplicator wins the Boolean existential k-pebble game on $E_{r}(F)$.

Proof: Let $\mathcal{A}$ be a winning strategy for the $(r, k)$-game on $F$. We first claim that we may assume without loss of generality that every extended partial truth assignment $(D, f)$ in $\mathcal{A}$ is such that if $(x, C) \in D$ and $(y, C) \in D$ for some $C \neq 1$, then $x=y$. Indeed, let $\mathcal{A}^{\prime}$ be the set of all extended partial truth assignments that are obtained from those in $\mathcal{A}$ in the following way: Let $a=(D, h)$ be an extended partial truth assignment in $\mathcal{A}$, where

$$
\begin{aligned}
D= & \left\{\left(x_{1,1}, C_{1}\right), \ldots,\left(x_{1, r_{1}}, C_{1}\right), \ldots,\right. \\
& \left(x_{s, 1}, C_{s}\right), \ldots,\left(x_{s, r_{s}}, C_{s}\right), \\
& \left.\left(x_{1}, 1\right), \ldots,\left(x_{q}, 1\right)\right\}
\end{aligned}
$$

with $C_{1}, \ldots, C_{s} \neq 1$. For each choice of $i_{1} \in\left\{1, \ldots, r_{1}\right\}, \ldots, i_{s} \in\left\{1, \ldots, r_{s}\right\}$, obtain an extended partial truth assignment $a^{\prime}=\left(D^{\prime}, h\right)$, where

$$
D^{\prime}=\left\{\left(x_{1, i_{1}}, C_{1}\right), \ldots,\left(x_{s, i_{s}}, C_{s}\right),\left(x_{1}, 1\right), \ldots,\left(x_{q}, 1\right)\right\},
$$

and put all these extended partial truth assignments in $\mathcal{A}^{\prime}$. It is not hard to see that $\mathcal{A}^{\prime}$ is also a winning strategy for the $(r, k)$-game on $F$.

Now, let $a=(D, f)$ be an extended partial truth assignment. We define an ordinary partial truth assignment $t_{a}$ as follows:

(i) The domain of $t_{a}$ is the set of all $x \in V$ such that $(x, C) \in D$ for some $C \in F \cup\{1\}$, together with all variables $y_{C, j}$ such that $(x, C) \in D$ for some $x \in V$ and $C \in F$.

(ii) If $(x, C) \in D$ for some $C \in F \cup\{1\}$, then $t_{a}(x)=f(x)$.

(iii) If $(x, C) \in D$ for some $C \in F$, let $l_{i}$ be the literal of $C=\left\{l_{1}, \ldots, l_{w}\right\}$ corresponding to variable $x$ and set $t_{a}\left(y_{C, j}\right)=0$ if $j<i$ and $t_{a}\left(y_{C, j}\right)=1$ if $j \geq i$ (here is where we use the assumption about the uniqueness of $x$ ).

First notice that each $t_{a}$ is a partial truth assignment to the variables of $E_{r}(F)$ that does not falsify any clause from $E_{r}(F)$. Moreover, if $a \subseteq b$, then $t_{a} \subseteq t_{b}$. Now, we construct our winning strategy $\mathcal{H}$ by including, for every $a \in \mathcal{A}$, every partial truth assignment $f$ such that $f \subseteq t_{a}$ and $|\operatorname{Dom}(f)| \leq k$. Conditions (i) and (ii) in Definition 2 are obviously satisfied. Let us consider condition (iii). Let $f \in \mathcal{H}$ be such that $|\operatorname{Dom}(f)| \leq k-1$. Then, there exists $a \in \mathcal{A}$ such that $f \subseteq t_{a}$. Since $|\operatorname{Dom}(f)| \leq k-1$, there exists a projection $b \subseteq a$ such that $|\operatorname{Dom}(b)| \leq k-1$ and $f \subseteq t_{b}$. Let $b=(D, g)$. Let $x$ be an initial variable. By the third property of a strategy for the extended game, there is an $h$ such that $g \subseteq h$ and $(D \cup\{(x, 1)\}, h) \in \mathcal{A}$. Thus $f \subseteq t_{b} \subseteq t_{c}$ where $c=(D \cup\{(x, 1)\}, h)$. Then, the projection of $t_{c}$ to the variables in $\operatorname{Dom}(f) \cup\{x\}$ is an extension of $f$ that belongs to $\mathcal{H}$ and has $x$ in its domain. Now let $y_{C, j}$ be an extension variable of clause $C$ with $|C|>r$. We have to consider two cases: (1) $(x, C) \in D$ for some $x$, and (2) otherwise. In case (1), the projection of $t_{b}$ to the variables in $\operatorname{Dom}(f) \cup\left\{y_{C, j}\right\}$ is an extension of $f$ that belongs to $\mathcal{H}$ and has $y_{C, j}$ in its domain. In case (2), there exists some variable $x$ and $h$ such that $g \subseteq h$ and $(D \cup\{(x, C)\}, h) \in \mathcal{H}$. This time $f \subseteq t_{c}$ where $c=(D \cup\{(x, C)\}, h)$, and the projection of $t_{c}$ to the variables in $\operatorname{Dom}(f) \cup\left\{y_{C, j}\right\}$ is an extension of $f$ that belongs to $\mathcal{H}$ and has $y_{C, j}$ in its domain. 
There is a strong reason to claim that the definition of the $(r, k)$-game is not arbitrary. Indeed, a sharp converse to Lemma 7 holds as we show next. Since this is stated here only for completeness and will not be used in what follows, we only provide a sketch of the proof.

Lemma 8 If the Duplicator wins the Boolean existential $(k+3)$-pebble game on $E_{r}(F)$, then the Duplicator wins the $(r, k)$-game on $F$.

Proof sketch: Suppose that the Duplicator wins the Boolean existential $(k+3)$-pebble game on $E_{r}(F)$. We describe a winning strategy for the Dupicator in the $(r, k)$-game on $F$. In order to decide her answers in the extended game on $F$, the Duplicator will play a Boolean game on $E_{r}(F)$ on the side in such a way that if the current extended partial truth assignment in the extended game is $(D, f)$, then the current partial truth assignment in the side game will be $f$. Note that $|\operatorname{Dom}(f)| \leq k \leq k+3$ and $f$ does not falsify any clause from $F$, so it does not falisfy any clause from $E_{r}(F)$ either. In the course of the play in the extended game, if the Spoiler restricts his current extended partial truth assignment to a subset $E \subseteq D$ (as in property (ii) in the definition of the extended game), then the Duplicator answers simply as she would answer in the side game on $E_{r}(F)$ if the Spoiler restricted his current partial truth assignment $f$ to the variables in $E$. If in the extended game the Spoiler requires the Duplicator to extend the current extended partial truth assignment $(D, f)$ with $|D|<k$ to a variable $x$ (as in property (iii) in the definition of the extended game), then the Duplicator answers simply as she would answer in the side game on $E_{r}(F)$ if the Spoiler required her to extend $f$ to the variable $x$. Finally, if the Spoiler requires the Duplicator to extend the current extended partial truth assignment $(D, f)$ with $|D|<k$ to satisfy a clause $C=l_{1} \vee \cdots \vee l_{w}$ of length at least $r+1$ (as in property (iv) in the definition of the extended game), then the Duplicator answers as follows: using the three remaining pebbles in side game, let us pretend that the Spoiler pebbles $y_{C, 0}$, $\operatorname{var}\left(l_{1}\right)$, and $y_{C, 1}$. If the answer of the Duplicator does not satisfy $l_{1}$, let us pretend that the Spoiler lifts the two pebbles in $y_{C, 0}$ and $\operatorname{var}\left(l_{1}\right)$ and puts them in $\operatorname{var}\left(l_{2}\right)$ and $y_{C, 2}$. Again, if the answer of the Duplicator does not satisfy $l_{2}$, let the Spoiler lift the two pebbles in $y_{C, 1}$ and $\operatorname{var}\left(l_{2}\right)$ and put them in $\operatorname{var}\left(l_{3}\right)$ and $y_{C, 3}$. By repeating this process, the Duplicator will eventually reach an extension of $f$ that sets some $l_{i}$ to true; otherwise some clause in $E_{r}(C)$ would eventually be falsified. This gives her the answer in the extended game: extend $D$ to $D \cup\left\{\left(\operatorname{var}\left(l_{i}\right), C\right)\right\}$ and $f$ to $f \cup\left\{\left(\operatorname{var}\left(l_{i}\right), a\right)\right\}$, where $a=1$ if $l_{i}$ is positive and $a=0$ if $l_{i}$ is negative. This completes the strategy of the Duplicator.

The result of this lemma means that we loose essentially nothing in restricting ourselves to playing the modified game on $E_{r}(F)$. We illustrate its use for the set of clauses expressing the dense linear order principle which says that a finite linear order cannot be dense.

For every $i, j \in\{1, \ldots, n\}$, let $x_{i, j}$ be a propositional variable whose intended meaning is that $i$ is smaller than $j$ in the linear ordering. For every $i, j, k \in\{1, \ldots, n\}$, let $z_{i, j, k}$ be a propositional variable whose intended meaning is that $i$ is smaller than $j$, and $j$ is smaller than $k$ in the linear ordering. The clauses of $D L O_{n}$ are the following:

$$
\begin{aligned}
& \neg x_{i, j} \vee \neg x_{j, i} \\
& x_{i, j} \vee x_{j, i} \\
& \neg x_{i, j} \vee \neg x_{j, k} \vee x_{i, k} \\
& \neg x_{i, j} \vee \neg x_{j, k} \vee z_{i, j, k} \\
& \neg z_{i, j, k} \vee x_{i, j} \\
& \neg z_{i, j, k} \vee x_{j, k} \\
& \neg x_{i, k} \vee z_{i, 1, k} \vee \cdots \vee z_{i, n, k} \quad\left(D_{i, k}\right)
\end{aligned}
$$


where $i, j, k \in\{1, \ldots, n\}$ and $i \neq j$ in (2). Since $D L O_{n}$ has large clauses, we employ the $(r, k)$-game introduced above.

Lemma 9 The Duplicator wins the extended $(3, n / 3)$-game on $D L O_{n}$, and therefore, every resolution refutation of $E_{3}\left(D L O_{n}\right)$ requires width $n / 3$.

Proof: For every linear ordering $\prec$ on $\{1, \ldots, n\}$, let $a_{\prec}=(E, g)$ be the extended partial truth assignment with

$$
\begin{aligned}
& E=E_{1} \cup E_{2} \cup E_{3} \cup E_{4} \\
& E_{1}=\left\{\left(x_{i, j}, 1\right): 1 \leq i, j \leq n\right\} \\
& E_{2}=\left\{\left(z_{i, j, k}, 1\right): 1 \leq i, j, k \leq n\right\} \\
& E_{3}=\left\{\left(x_{i, j}, D_{i, j}\right): j \prec i\right\} \\
& E_{4}=\left\{\left(z_{i, j, k}, D_{i, k}\right): i \prec j \prec k\right\} .
\end{aligned}
$$

The mapping $g$ is defined as $g\left(x_{i, j}\right)=1$ if $i \prec j$ and 0 otherwise, and $g\left(z_{i, j, k}\right)=1$ if $i \prec j \prec k$ and 0 otherwise. During this proof, the set $E$ in $a_{\prec}=(E, g)$ is called the domain of $a_{\prec}$. By the way it is defined, $a_{\prec}$ is an extended partial truth assignment.

We define our winning strategy $\mathcal{A}$ as the set containing every extended partial truth assignment $(D, f) \subseteq$ $a_{\prec}$ with $|D| \leq n / 3$ for every linear ordering $\prec$ on $\{1, \ldots, n\}$. Thus, $\mathcal{A}$ satisfies conditions (i) and (ii) of extended winning strategy. We will show that condition (iii) is also satisfied. Let $(D, f) \subseteq a_{\prec}$ be any element of $\mathcal{A}$ with $|D|<n / 3$. For any $x_{i, j}$, the pair $\left(x_{i, j}, 1\right)$ is in the domain of $a_{\prec}$, and consequently, the projection of $a_{\prec}$ to $D \cup\left\{\left(x_{i, j}, 1\right)\right\}$ belongs to $\mathcal{A}$. Analogously, for every $z_{i, j, k}$, the pair $\left(z_{i, j, k}, 1\right)$ is in the domain of $a_{\prec}$, and consequently, the projection of $a_{\prec}$ to $D \cup\left\{\left(z_{i, j, k}, 1\right)\right\}$ is also in $\mathcal{A}$.

Let us consider now condition (iv). Let $(D, f) \subseteq a_{\prec}$ be any element of $\mathcal{A}$ with $|D|<n / 3$, and let $C$ be a clause of $E_{3}\left(D L O_{n}\right)$ of length at least 4 . Such a $C$ is a clause of type (7) in $D L O_{n}$, say $C=D_{i, k}$, since these are the only clauses of length more than three. Let $N$ be the set of indices in $\{1, \ldots, n\}$ that are referenced in $D$. That is, $N$ contains $i^{\prime}$ and $j^{\prime}$ if $\left(x_{i^{\prime}, j^{\prime}}, C^{\prime}\right)$ is in $D$ for some $C^{\prime}$, and $N$ contains $i^{\prime}, j^{\prime}$, and $k^{\prime}$ if $\left(z_{i^{\prime}, j^{\prime}, k^{\prime}}, C^{\prime}\right)$ is in $D$ for some $C^{\prime}$. Since $|D|<n / 3$, we have $|N| \leq n-3$. Let $1 \leq i^{\prime}, j^{\prime} \leq n$ be an arbitrary pair of indices from $\{1, \ldots, n\}$. We will show that there exists a linear order $\prec^{\prime}$ on $\{1, \ldots, n\}$ such that (1) $\prec$ and $\prec^{\prime}$ coincide on $N$, i.e., $i^{\prime} \prec j^{\prime}$ iff $i^{\prime} \prec^{\prime} j^{\prime}$ for every $i^{\prime}, j^{\prime} \in N$, and (2) the domain of $a_{\prec^{\prime}}$ contains $\left(x, D_{i, k}\right)$ for some $x$. Thus, the projection of $a_{\prec^{\prime}}$ to $D \cup\left\{\left(x, D_{i, k}\right)\right\}$ belongs to $\mathcal{A}$. To construct $\prec^{\prime}$ we do the following: if $i$ and $k$ belong to $N$ and $i \prec k$, then we fix $\prec^{\prime}$ to be a linear ordering that coincides with $\prec$ on $N$ and such that $i \prec^{\prime} j \prec^{\prime} k$ for some $j$ not in $N$. It is immediate to see that such a $\prec^{\prime}$ exists and that $\left(z_{i, j, k}, D_{i, k}\right)$ belongs to the domain of $a_{\prec^{\prime}}$. Otherwise, we can find some linear ordering $\prec^{\prime}$ that coincides with $\prec$ on $N$ and such that $k \prec^{\prime} i$. In this case $\left(x_{i, k}, D_{i, k}\right)$ belongs to the domain of $a_{\prec^{\prime}}$ and we are done.

We stress on the fact that the introduction of the new game was motivated by an attempt to generalize the construction of winning strategies in the presence of auxiliary variables. A winning strategy for the Duplicator in the original game on the non-deterministic extension $E_{3}\left(D L O_{n}\right)$ could also be easily found directly.

To complete this section, in view of the width lower bound that we just proved, it is quite interesting that $D L O_{n}$ has a resolution refutation of polynomial size as we show next.

Theorem 4 The set of clauses $D L O_{n}$, and therefore also $E_{3}\left(D L O_{n}\right)$, has a resolution refutation of size $O\left(n^{4}\right)$. 
Proof: The idea of the proof is to derive the clauses

$$
D_{k}(i)=\neg x_{1, i} \vee z_{1,1, i} \vee \cdots \vee z_{1, k, i}
$$

for every $k \in\{1, \ldots, n\}$ and $i \in\{1, \ldots, n\}$. Once this is done, from $D_{1}(i)$ we obtain $\neg x_{1, i}$ for every $i \in\{1, \ldots, n\}$ by a cut with $\neg z_{1,1, i}$ which is derived from $\neg x_{1,1}$ and (5) (observe that $\neg x_{1,1}$ is simply (1) in the particular case $i=j=1$ ). Then we obtain $x_{i, 1}$ for every $i \in\{2, \ldots, n\}$ by a cut with (2), and $\neg z_{i, 1, k}$ for every $i, k \in\{1, \ldots, n\}$ by a cut with (6). Then we eliminate all occurrences of all variables $x_{i, 1}, x_{1, i}$ in $D L O_{n}$, and $z_{i, 1, k}$ from (7). The resulting formula would contain a copy of $D L O_{n-1}$ up to renaming of indices.

In order to derive $D_{k}(i)$, do the following. We proceed by reverse induction on $k$. For $k=n$, note that each $D_{n}(i)$ is an initial clause. Suppose now we have derived every $D_{k}(i)$ and we want to derive every $D_{k-1}(i)$. First, note that $D_{k-1}(k)$ is derived at once from $D_{k}(k)$ and $\neg z_{1, k, k}$, which is derived by a cut between (6) and $\neg x_{k, k}$ (again $\neg x_{k, k}$ is (1) in the particular case $i=j=k$ ). To derive $D_{k-1}(i)$ from $D_{k}(i)$ and $D_{k-1}(k)$ for $i \neq k$, we will derive the intermediate clauses

$$
E_{k-1}(j, i)=D_{k-1}(i) \vee z_{1, j, k} \vee \cdots \vee z_{1, k-1, k}
$$

for $j=1, \ldots, k$. Note that $E_{k-1}(k, i)$ is $D_{k-1}(i)$ because the range of the dots $\cdots$ is empty. Start with $D_{k}(i)$ and cut with (5) on $z_{1, k, i}$ to obtain $D_{k-1}(i) \vee x_{1, k}$. Then cut this with $D_{k-1}(k)$ on $x_{1, k}$ to obtain $E_{k-1}(1, i)$. Now we derive $E_{k-1}(j+1, i)$ from $E_{k-1}(j, i)$. Cut $E_{k-1}(j, i)$ with (6) on $z_{1, j, k}$ to obtain $E_{k-1}(j+1, i) \vee x_{j, k}$. Then cut this with (3) on $x_{j, k}$ to obtain

$$
E_{k-1}(j+1, i) \vee \neg x_{k, i} \vee x_{j, i}
$$

Separately, take $D_{k}(i)$ and cut with (6) on $z_{1, k, i}$ to obtain

$$
D_{k-1}(i) \vee x_{k, i}
$$

Then cut the last two displayed clauses on $x_{k, i}$ to obtain $E_{k-1}(j+1, i) \vee x_{j, i}$. Cut this with (4) on $x_{j, i}$ to obtain

$$
E_{k-1}(j+1, i) \vee \neg x_{1, j} \vee z_{1, j, i}
$$

Separately, cut $E_{k-1}(j, i)$ with (5) on $z_{1, j, k}$ to obtain $E_{k-1}(j+1, i) \vee x_{1, j}$. Finally, cut this with the last displayed clause to obtain $E_{k-1}(j+1, i)$. Note here that $z_{1, j, i}$ is absorved by $D_{k-1}(i)$ in $E_{k-1}(j+1, i)$. This completes the refutation of $D L O_{n}$. Its size $O\left(n^{4}\right)$ because it consists of $n$ phases of length $O\left(n^{3}\right)$ each.

Therefore, the dense linear order principle is another example of a tautology witnessing that the relationship between size and width of Ben-Sasson and Wigderson cannot be made subpolynomial. Indeed, $D L O_{n}$ has resolution proofs of size $O\left(n^{4}\right)$ yet it requires width $\Omega(n)$ to refute. A tighter lower bound to the size-width relationship was provided by Bonet and Galesi who showed that the minimum principle $G T_{n}$ has resolution proofs of size $O\left(n^{2}\right)$ yet it requires width $\Omega(n)$ to refute. We note that the width lower bound for $G T_{n}$ could also be derived using Lemma 7 and a game theoretic argument.

\section{Conclusions}

We have provided a charaterization of resolution width in terms of the existential pebble game which was introduced in the context of finite model theory. In that context, the game was used as a tool to obtain 
non-expressibility results for the existential positive fragment of infinitary logic with finitely many variables which subsumes Datalog. Our result indicates that there is a tight connection between both areas, and also with the area of the theory of constraint satisfaction problems since existential pebble games play an important role there $[20,21,13]$. We think it is worth exploring further these connections. In particular, it is an interesting project to try to establish a precise connection between definability in the existential positive fragment of infinitary logic with finitely many variables and the prover-liar games of Buss and Pudlák [23], and to interpret and use that correspondence in both fields. Also, this is a good time to mention Pudlák's view of resolution proofs as games [22]. His view is slightly more complicated than ours (with strategies and super-strategies), but is essentially equivalent if we restrict the size of the records to $k$ in his game. The formulation with pebble games has the benefit of being of more algebraic nature, and of establishing the link with finite model theory.

Our results may also have some impact on the algorithmic aspects of resolution that we discuss next. From the known results in the area of constraint satisfaction problems, it is not hard to prove that the Duplicator wins the existential $k$-pebble game on structures $\mathbf{A}$ and $\mathbf{B}$ if and only if every structure of treewidth less than $k$ that maps homomorphically to $\mathbf{A}$ also maps homomorphically to $\mathbf{B}$ [16]. In view of our results, this is interpreted as follows in the context of resolution: a 3-CNF formula $F$ requires resolution width $w$ if and only if every 3-CNF formula $G$ of tree-width less than $w$ that maps homomorphically to $F$ is satisfiable. This explains why formulas of small tree-width have small resolution proofs and sheds some more light on the recent algorithmic results of Alekhnovich and Razborov [2]. We believe these connections should be explored further.

Acknowledgments. We thank an anonymous referee for very detailed comments that improved the presentation.

\section{References}

[1] M. Alekhnovich, E. Ben-Sasson, A. Razborov, and A. Wigderson. Space complexity in propositional calculus. SIAM Journal of Computing, 31(4):1184-1211, 2002. A preliminary version appeared in STOC 2000.

[2] M. Alekhnovich and A. Razborov. Satisfiability, branch-width and Tseitin tautologies. In 43rd Annual IEEE Symposium on Foundations of Computer Science, pages 593-603, 2002.

[3] M. Alekhnovich and A. A. Razborov. Resolution is not automatizable unless W[P] is tractable. In 42nd Annual IEEE Symposium on Foundations of Computer Science, pages 210-219, 2001.

[4] M. Alekhovich, E. Ben-Sasson, A. A. Razborov, and A. Wigderson. Pseudorandom generators in propositional proof complexity. SIAM Journal on Computing, 34(1):67-88, 2004. A preliminary version appeared in FOCS 2000.

[5] A. Atserias. Unsatisfiable random formulas are hard to certify. Journal of the ACM, 51(2):281-311, 2004. A preliminary version appeared in LICS 2002.

[6] A. Atserias and M. L. Bonet. On the automatizability of resolution and related propositional proof systems. Information and Computation, 189(2):182-201, 2004. A preliminary version appeared in CSL 2002.

[7] P. Beame and T. Pitassi. Simplified and improved resolution lower bounds. In 37th Annual IEEE Symposium on Foundations of Computer Science, pages 274-282, 1996. 
[8] E. Ben-Sasson and N. Galesi. Space complexity of random formulas in resolution. Random Structures and Algorithms, 23(1):92-109, 2003. A preliminary version appeared in CCC 2001.

[9] E. Ben-Sasson and A. Wigderson. Short proofs are narrow-resolution made simple. Journal of the ACM, 48(2):149-169, 2001. A preliminary version appeared in STOC 1999.

[10] M. L. Bonet and N. Galesi. Optimality of size-width trade-offs for resolution. Computational Complexity, 10(4):261-276, 2001. A preliminary version appeared in FOCS 1999.

[11] V. Chvátal and E. Szemerédi. Many hard examples for resolution. Journal of the ACM, 35(4):759-768, 1988.

[12] M. Clegg, J. Edmonds, and R. Impagliazzo. Using the Groebner basis algorithm to find proofs of unsatisfiability. In 27th Annual ACM Symposium on the Theory of Computing, 1995.

[13] V. Dalmau, P. G. Kolaitis, and M. Y. Vardi. Constraint satisfaction, bounded treewidth, and finite variable logics. In 8th International Conference on Principles and Practice of Constraint Programming (CP), volume 2470 of Lecture Notes in Computer Science, pages 310-326. Springer, 2002.

[14] H. Ebbinghaus and J. Flum. Finite Model Theory. Springer-Verlag, 1995.

[15] J. L. Esteban and J. Torán. Space bounds for Resolution. Information and Computation, 171(1):84-97, 2001. A preliminary version appeared in STACS 1999.

[16] T. Feder and M. Y. Vardi. The computational structure of monotone monadic SNP and constraint satisfaction: A study through Datalog and group theory. SIAM Journal of Computing, 28(1):57-104, 1998. A preliminary version appeared in STOC 1993.

[17] M. Grohe. Finite variable logics in descriptive complexity theory. Bulletin of Symbolic Logic, 4:345398, 1998.

[18] A. Haken. The intractability of resolution. Theoretical Computer Science, 39:297-308, 1985.

[19] P. G. Kolaitis and M. Y. Vardi. On the expressive power of Datalog: tools and a case study. Journal of Computer and System Sciences, 51:110-134, 1995.

[20] P. G. Kolaitis and M. Y. Vardi. Conjunctive-query containment and constraint satisfaction. Journal of Computer and System Sciences, 61(2):302-332, 2000. A preliminary version appeared in PODS 1998.

[21] P. G. Kolaitis and M. Y. Vardi. A game-theoretic approach to constraint satisfaction. In 7th National Conference on Artificial Intelligence, pages 175-181, 2000.

[22] P. Pudlák. Proofs as games. American Mathematical Monthly, June-July:541-550, 2000.

[23] P. Pudlák and S. R. Buss. How to lie without being (easily) convicted and the lengths of proofs in propositional calculus. In Pacholski and Tiuryn, editors, Computer Science Logic '94, volume 933 of Lecture Notes in Computer Science, pages 151-162. Springer-Verlag, 1995.

[24] R. Raz. Resolution lower bounds for the weak pigeonhole principle. Journal of the ACM, 51(2): 115-138, 2004. A preliminary version appeared in STOC 2002.

[25] A. A. Razborov. Resolution Lower Bounds for the Weak Functional Pigeonhole Principle, Theoretical Computer Science, 303(1):233-243, 2003. 
[26] A. A. Razborov. Resolution lower bounds for perfect matching principles. Journal of Computer and System Sciences, 69(1):3-27, 2004. A preliminary version appeared in CCC 2002.

[27] S. Riis. A complexity gap for tree-resolution. Computational Complexity, 10:179-209, 2001.

[28] J. Torán. Lower bounds for space in Resolution. In Computer Science Logic '1999, 13th Annual Conference of the EACSL, Lecture Notes in Computer Science. Springer-Verlag, 1999.

[29] A. Urquhart. Hard examples for resolution. Journal of the ACM, 34(1):209-219, 1987. 